\title{
ZFP36 stabilizes RIP1 via degradation of XIAP and CIAP2 thereby promoting ripoptosome assembly
}

\author{
Tommaso Selmi ${ }^{1}$, Claudia Alecci ${ }^{1}$, Miriam dell' Aquila ${ }^{1}$, Lucia Montorsi ${ }^{1}$, Andrea Martello ${ }^{1}$, Filippo Guizzetti ${ }^{1}$, \\ Nicola Volpi ${ }^{1}$, Sandra Parenti ${ }^{1}$, Sergio Ferrari ${ }^{1}$, Paolo Salomoni ${ }^{2}$, Alexis Grande ${ }^{1}$ and Tommaso Zanocco-Marani ${ }^{*}$
}

\begin{abstract}
Background: ZFP36 is an mRNA binding protein that exerts anti-tumor activity in glioblastoma by triggering cell death, associated to an increase in the stability of the kinase RIP1.

Methods: We used cell death assays, size exclusion chromatography, Co-Immunoprecipitation, shRNA lentivectors and glioma neural stem cells to determine the effects of ZFP36 on the assembly of a death complex containing RIP1 and on the induction of necroptosis.

Results: Here we demonstrate that ZFP36 promotes the assembly of the death complex called Ripoptosome and induces RIP1-dependent death. This involves the depletion of the ubiquitine ligases CIAP2 and XIAP and leads to the association of RIP1 to caspase-8 and FADD. Moreover, we show that ZFP36 controls RIP1 levels in glioma neural stem cell lines.

Conclusions: We provide a molecular mechanism for the tumor suppressor role of ZFP36, and the first evidence for Ripoptosome assembly following ZFP36 expression. These findings suggest that ZFP36 plays an important role in RIP1-dependent cell death in conditions where IAPs are depleted.
\end{abstract}

Keywords: ZFP36, Ripoptosome, XIAP, cIAP2, RIP1, Glioma, Necroptosis

\section{Background}

ZFP36 belongs to the TIS11/TTP protein family, a class of mRNA binding proteins carrying a conserved zinc finger domain, which binds to AU-rich elements in the 3' UTR of mRNAs. ZFP36 triggers mRNA decay and or inhibition of translation of its targets, resulting in negative regulation of gene expression [1]. A growing body of literature demonstrates the ability of ZFP36 to negatively regulate mRNAs coding for oncogenes in different cellular contexts thereby exerting anti-tumor activities [2-5]. Interestingly, it has been shown that ZFP36 sensitizes transformed cell lines to TNF alpha-induced cell death [6] and we have recently described the role of ZFP36 in promoting necroptosis in glioma cell lines [4].

Cells die by activating many forms of programmed cell death, such as apoptosis and necroptosis, which are characterized by the assembly of intracellular complexes

\footnotetext{
* Correspondence: zanocco@unimore.it

${ }^{1}$ Department of Life Sciences, University of Modena and Reggio Emilia, Modena 41125, Italy

Full list of author information is available at the end of the article
}

that execute death sentences [7]. The Ripoptosome is a recently described death-inducing complex, whose assembly is triggered in responsive cancer cell lines by Etoposide or SMAC-mimetics [8, 9]. Once assembled, this complex signals both apoptotic and necroptotic cell death, independently of the activation of the TNFreceptor superfamily [8]. The Ripoptosome contains the core components RIP1, caspase- 8 and FADD. It has been suggested that the stability of this complex is regulated by the E3 ubiquitine ligases XIAP, CIAP1 and cIAP2, which act by marking the core components for proteasome-mediated degradation [8]. Etoposide and Interferons are used to induce cell death dependent on RIP1 [8, 10] however, only few details are known regarding the intracellular mediators of the necroptotic effects of these drugs [11]. We previously demonstrated that ZFP36 acts as tumor suppressor in glioma cell lines by inducing necroptotic cell death and we speculated that RIP1 stabilization could be responsible for the execution of this death program [4]. Here we show that ZFP36 stabilizes RIP1, promotes the assembly of the Ripoptosome 
and induces RIP1-dependent cell death. The key molecular event underlining this process is the depletion of the IAP proteins XIAP and cIAP2, which we and others $[4,12]$ previously described as a target of ZFP36mediated mRNA decay. Finally we demonstrate that loss of ZFP36 in glioma cancer stem cells leads to the downregulation of RIP1, thus reinforcing the molecular link between ZFP36 and RIP1.

\section{Methods}

\section{Cell cultures}

Human embryonic kidney (HEK) $293 \mathrm{~T}$ cells were obtained from ATCC and cultured in IMDM medium (Euroclone) supplemented with $10 \%$ heat-inactivated fetal bovine serum (Biowhittaker), $1 \mathrm{mM}$ L-glutamine and penicillin/streptomycin $(100 \mu \mathrm{g} / \mathrm{ml})$ (Euroclone). As described by Pollard et al. [13], G144 human glioma neural stem (GNS) cell lines were maintained onto laminin $(10 \mathrm{mg} / \mathrm{ml}$, Sigma) coated dishes in expansion media (RHA-B media from Stem Cell Technologies) supplemented with $20 \mathrm{ng} / \mathrm{mL}$ of EGF (recombinant mouse, PeproTech 315-09 $20 \mathrm{ng} / \mathrm{ml}$ ) and $20 \mathrm{ng} / \mathrm{mL}$ bFGF (recombinant human, PeproTech100-18B, $20 \mathrm{ng} / \mathrm{ml}$ ). Medium was changed every 2 days, and cells were split 1:2 to $1: 3$ once culture became confluent.

\section{Chemicals and treatments}

Where indicated, HEK293T were treated with $100 \mu \mathrm{M}$ Etoposide (Invitrogen), $20 \mu \mathrm{M}$ Z-VAD-FMK (Invitrogen), $50 \mu \mathrm{M}$ Necrostatin-1 (Enzo Life Sciences) and DMSO (Sigma) served was administered as vehicle.

\section{Expression constructs}

Full-length ZFP36 was cloned in the pCDNA3.1 vector (Invitrogen) as already described in reference 4. The pCI-IRES-HA-XIAP ( $\triangle 3^{\prime}$ UTR XIAP) allows the expression of the XIAP coding sequence driven by its own IRES and was a gift from Dr Salomoni.

\section{Cell death assays}

HEK293T were transfected using the Transit 2020 Reagent (Mirus Bio LLC) according to manufacturer's instruction. In the co-transfection experiments, the same amount in $\mu \mathrm{g}$ for each plasmid was added to the transfection mix. 48 hours after transfection, cell death was assessed by Propidium Iodide (PI) or Annexin V/Propidium Idodide staining (AV/PI). For PI staining, cells were cultured in the presence of $0.5 \mu \mathrm{g} / \mathrm{mL}$ PI (BD PharMingenTM) for 15 minutes and imaged using a Leica 5515015 inverted microscope. AnnexinV/PI positivity was assessed by BD PharMingenTM kit (cat. Number 556547) at the cytofluorimeter (Coulter Epics XL MCL, Beckman Coulter Inc.). Trypan Blue positivity was used to count dead cells in Neubauer Chambers. HEK293 cells were gently detached from plates after short incubations (60") in 1X Trypsin-EDTA solution (Life Technologies). Error bars represent mean and SEM calculated on a set of three to four independent experiments (" $\mathrm{p}<0.05$; **" $\mathrm{p}<0.01)$.

\section{Chromatographic separation}

Cellular lysates were fractionated by gel-permeation sizeexclusion chromatography on Sepharose 6B (Sigma-Aldrich, fractionation range for globular proteins $1 \times 10^{4} \div$ $4 \times 10^{6}$ ) column of $90 \mathrm{~cm}$ high and $1 \mathrm{~cm}$ of diameter. The column was eluted with $5 \% \mathrm{w} / \mathrm{v}$ sucrose, $0.1 \% \mathrm{w} / \mathrm{v}$ CHAPS, $20 \mathrm{mM}$ HEPES, $5 \mathrm{mM}$ DTT, and $50 \mathrm{mM} \mathrm{NaCl}$, buffered at $\mathrm{pH}$ 7.0. Column flow was $1 \mathrm{~mL} / 5 \mathrm{~min}$ and fractions volume was set at $2 \mathrm{~mL}$. The column was calibrated with protein standards and blue dextran (SigmaAldrich). After elution, fractions were freeze-dried and further analyzed.

\section{Co-Immunoprecipitations, western blots and antibodies}

For the Ripoptosome immunoprecipitation, cells were transfected with the ZFP36 expression vector or with the empty vector. 24 hours following transfection, Z-VADFMK and, where indicated, also Etoposide were added for further 24 hours. Cells were then lysed in $30 \mathrm{mM}$ Tris (pH 7.4), $120 \mathrm{mM} \mathrm{NaCl}, 2 \mathrm{mM}$ EDTA, $2 \mathrm{mM} \mathrm{KCl}, 10 \%$ glycerol, $0.2 \% \mathrm{NP}-40,1 \mathrm{mM}$ sodium deoxycholate, $1 \mathrm{mM}$ sodium orthovanadate and Complete Protease Inhibition Cocktail (Roche), and caspase- 8 was immunoprecipitated with $2 \mathrm{mg}$ of caspase-8 (1C12) monoclonal antibody (Cell Signaling, 9746) coupled to protein $\mathrm{G}$ agarose (KPL). The CO-IP negative control was immunoprecipitated using non-specific mouse IgG (Santa Cruz Biotechnology). Immunoprecipitates where resuspended in 2x Laemmli Buffer without SDS and heated at $100^{\circ}$ for $5^{\prime}$ prior to loading onto SDS-polyacrylamide gels. Membranes were probed against RIP1 (BD Transduction Laboratories, \#610458), caspase-8 (1C12) (Cell Signaling, 9746) and (BD Transduction Laboratories, \#610399). IgG-HRP secondary antibodies were from Santa Cruz Biotechnology (sc-2031) and the Mouse TrueBlot (ebioscience).

Other antibodies used were: ZFP36 (Abcam, ab124024), TTP (H-120) (Santa Cruz biotecnology, sc-8458), XIAP (Cell Signaling, \#2042), cIAP2 (H-85) sc-7944 (Santa Cruz Biotchnology), PARP-1 (F-2) sc-8007 (Santa Cruz biotecnology), MLKL M6697 Sigma-Aldrich), VINCULIN clone V284 (Merck Millipore). Expression of actin was analyzed with a mouse anti-human pan-actin MoAb (SigmaAldrich) to normalize protein samples.

\section{Lentivectors}

To knockdown ZFP36 expression in human glioma neural stem cells, we used the commercially available pGIPZ-lentiviral shRNAmir vectors containing a hairpin 


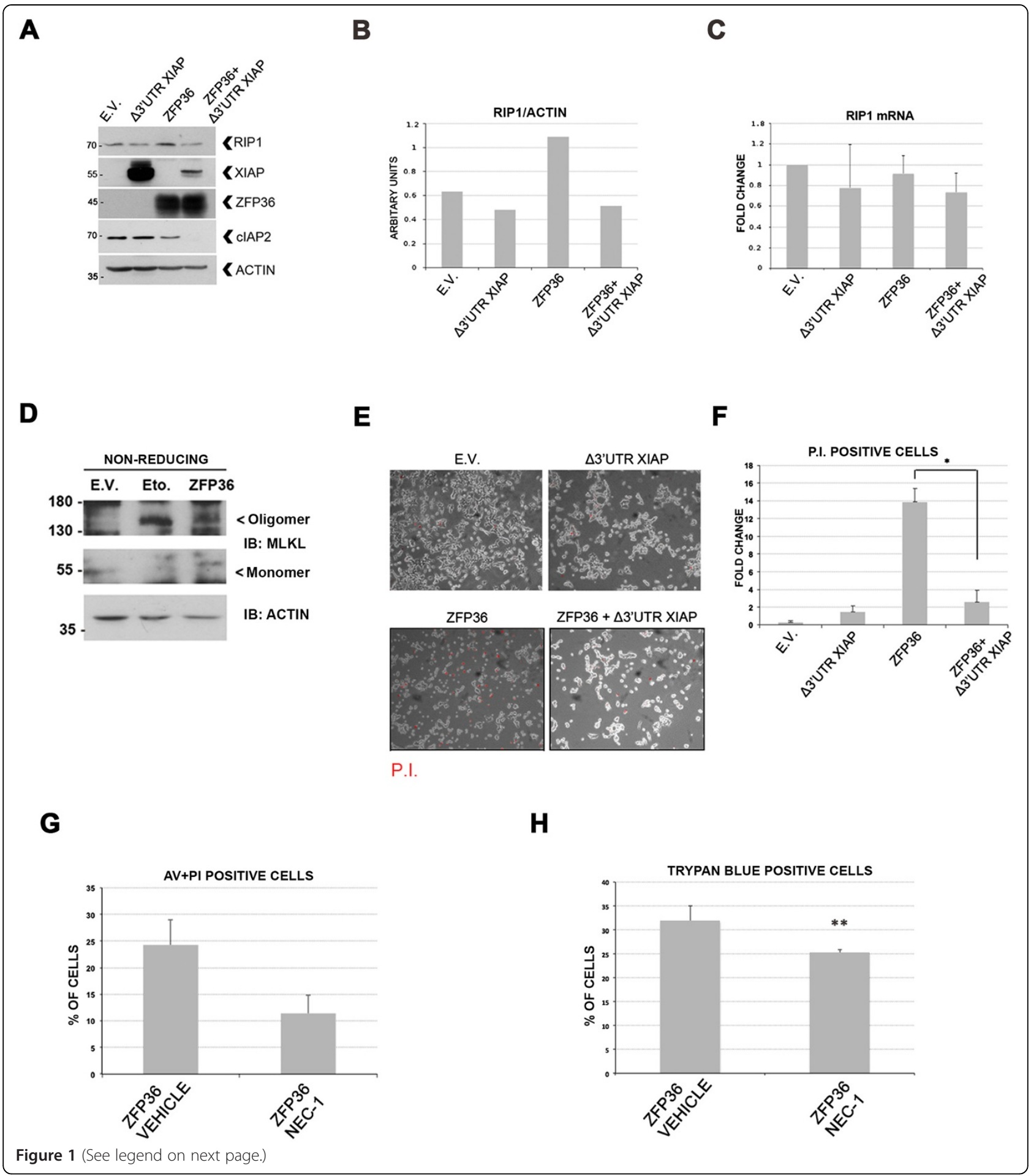


(See figure on previous page.)

Figure 1 ZFP36 controls RIP1 stability by depleting IAPs and promotes RIP1-dependent cell death. A). Transfection of HEK293T cells with a ZFP36 construct (ZFP36) correlates with the depletion of CIAP2 and with an increase in the stability of RIP1 at 48 hours. HEK293T cells transfected with a XIAP construct depleted of its own $3^{\prime} U T R\left(\triangle 3^{\prime} U T R\right.$ XIAP) show a reduction in the levels of RIP1, even in the presence of ZFP36 (ZFP36 + $\triangle 3^{\prime} U T R$ XIAP). B) Image J software was used to normalize the levels of RIP1 over beta-ACTIN. C) Real Time PCR showing RIP1 mRNA levels following ZFP36 and $\triangle 3^{\prime} U T R$ XIAP over-expression. D) Both the transfection of ZFP36 and the treatment with Etoposide induce the formation of MLKL homo-oligomers and a decrease of MLKL monomers. E.V. cells were incubated with DMSO as this is the vehicle for Nec-1 administration. Non-reducing conditions were applied in order to preserve intact oligomers of MLKL. E) HEK293T transfected with the ZFP36 construct (ZFP36) show increased positivity to Propidium lodide staining (P.I.), which is reduced in the presence of the $\Delta 3^{\prime} U T R$ XIAP construct (ZFP36+ $\triangle 3^{\prime}$ UTR XIAP). F) Percentage of P.I. positive cells obtained from 5 fields for each condition from experiment 1C. G-H) 48 hours treatment with $50 \mu \mathrm{M}$ Necrostatin-1 inhibits ZFP36-dependent cell death (ZFP36 Nec-1) if compared to DMSO treated cells (ZFP36 vehicle), as assessed by cytofluorimetric analysis of Annexin V / P.I. or positivity to Trypan blue. The graphs in this figure represent the mean and SEM calculated on a set of three to four independent experiments $\left({ }^{*} p<0.05 ;{ }^{*} p<0.01\right)$.

sequence targeting ZFP36 (Open Biosystems). The hairpin sequence was as follows: CGACTTTATTTATTCT AATATTACATCTGTGGCTTCACTAATATTAGAATA AATAAAGTCG.

The shRNA-containing lentiviral vector (Open Biosystems) was cotransfected with lentiviral packaging constructs into HEK293T cells to produce shRNA-carrying lentivirus particles. Supernatants were collected at $48 \mathrm{~h}$ after transfection and viral particles were concentrated using PEG (System Biosciences). GNS cells were transduced $(\mathrm{MOI}=10)$ for $1 \mathrm{~h}$ and subsequently supplemented with fresh media. After expansion, cells were sorted (MoFlo cell sorter, Beckman Coulter Inc.) based on GFP expression values, obtaining two homogeneous populations expressing either the (pGIPZ) scrambled vector, or pGIPZ shRNA ZFP36 (shZFP36). To perform over-expression experiments, the coding sequence of ZFP36 [4] was cloned in the pRRLSIN.cPPT.PGKGFP.WPRE vector. The procedure for producing the lentiviral particles and infecting the cells was the same as described for the pGIPZ vectors. ZFP36 expressing cells were used for experiments 24 to 48 hours after infection.

\section{Results \\ ZFP36-mediated RIP1 stabilization depends on IAPs degradation and correlates with increased cell death}

Experimental evidences demonstrate that ZFP36 triggers the degradation of XIAP and cIAP2 mRNAs, which carry AU-rich elements in their 3'UTR [4, 12]. Figure 1A shows that in HEK293T cells, that endogenously express cIAP2 and lower levels of XIAP, ZFP36 over-expression determines the down-regulation of cIAP2, which correlates with an increase in RIP1 (Figure 1A, B). This confirms that loss of IAPs activity can stabilize RIP1 $[8,14]$ and suggests that ZFP36 might regulate RIP1 through IAPs degradation. Accordingly, the observed increase in RIP1 was abolished by the co-transfection of a XIAP construct lacking the 3' UTR, which cannot be degraded by ZFP36 ( $\triangle 3^{\prime}$ UTR XIAP) (Figure 1A, B). This was further confirmed when a more physiological induction of ZFP36 was achieved by means of lentiviral infection (results are shown in additional file $1 \mathrm{~A}, \mathrm{~B}$ ) in the supplementary material section. In order to verify whether the increase of RIP1 depends on protein stabilization rather than on increased mRNA expression, we analyzed by Real Time PCR the levels of RIP1 mRNA. No statistically significant changes in RIP1 mRNA levels were detected (Figure 1C), suggesting that RIP1 protein is stabilized following ZFP36 expression.

Mixed lineage kinase domain-like protein (MLKL) is currently the main confirmed effector of RIP kinasesdependent necropotsis [15]. Upon activation of necroptosis, the monomeric form of MLKL decreases, owing to the formation of homo-oligomers that relocate to the plasma and intracellular membranes, where they compromise membrane integrity, resulting in cell death [15]. As a result of ZFP36 overexpression, the monomeric form of MLKL decreases while an increase of the homooligomeric form is observed (Figure 1D).

As cells overexpressing ZFP36 show an increase in RIP1 and in the oligomerized MLKL, we checked the permeability of these cells to propidium iodide. As shown in Figure 1E-F, where graph $F$ recaps the percentages observed in 1E, cells overexpressing ZFP36 are more permeable to propidium iodide than cells expressing ZFP36 and the $\Delta 3$ 'UTR XIAP, suggesting that they are less sensitive to ZFP36-induced cell death (ZFP36 $+\Delta 3$ 'UTR XIAP). To confirm that ZFP36-induced cell death is dependent on RIP1 we inhibited this kinase with its inhibitor Necrostatin-1 (Nec-1). Figure 1G-H shows that Nec-1 partly restrains ZFP36-dependent cell death in HEK293T cells, thus confirming that ZFP36 triggers RIP1-dependent cell death. Collectively, the data shown in Figure 1 demonstrate that the negative regulation exerted by ZFP36 over the XIAP and cIAP2 E3 ubiquitine ligases is a key mechanism controlling the stability of RIP1 and the sensitivity to necroptotic cell death.

\section{ZFP36-mediated RIP1 stabilization leads to Ripoptosome assembly}

Based on these observations, we hypothesized that the increased availability of RIP1 could result in its loading into the 2MDa Ripoptosome. To assess this, we first confirmed 
the presence of RIP1 in high molecular weight complexes only in the presence of ZFP36 (Figure 2A) and then we co-immunoprecipitated the Ripoptosome components (Figure 2B-D). Our results demonstrate that the binding of RIP1 to caspase-8 is promoted by ZFP36 overexpression (Figure $2 \mathrm{~B}, \mathrm{C}$ ), and that similar results are observed in response to Etoposide (Eto.), a Ripoptosome-stabilizing drug that acts through depleting IAPs [8]. When comparing empty vector (E.V.) to ZFP36-transfected cells (ZFP36), the amount of RIP1 bound to caspase- 8 was strongly reduced (Figure 2C) concomitantly to increased association between caspase-8 and FADD (Figure 2D). This suggests that ZFP36 determines, by stabilizing RIP1, the aggregation of a complex which, according to published data [11, 16, 17], might unbalance cells towards necroptosis rather than apoptosis. Therefore, we conclude that ZFP36 controls RIP1 stability, promotes its assembly in a high molecular weight complex and enhances its association to caspase-8 and FADD.

A
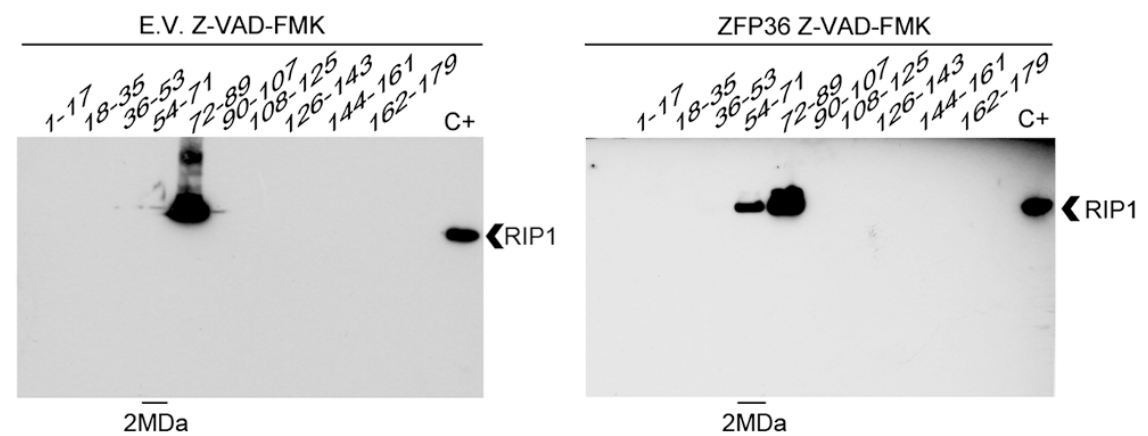

B
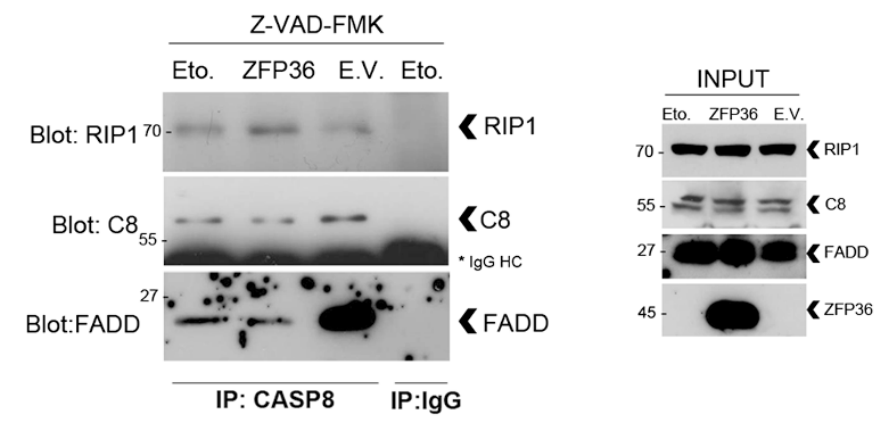

C

D
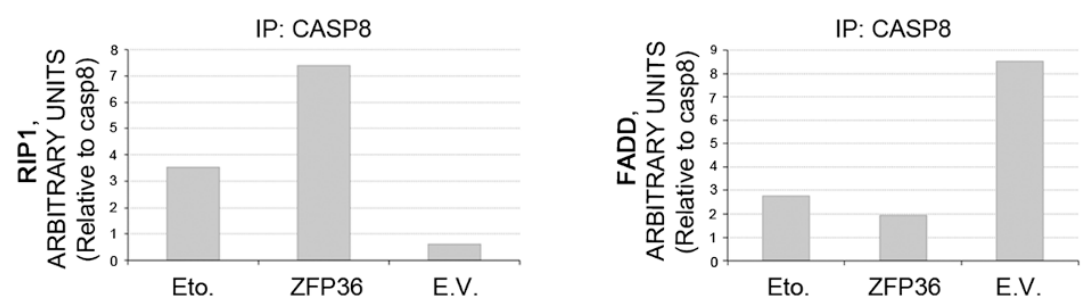

Figure 2 ZFP36 promotes RIP1 assembly in a high molecular weight complex containing caspase 8 and FADD. A, B) Following 24 hours of empty vector/ZFP36 transfection, HEK293T cells were further treated for 24 hours in the presence of $20 \mu \mathrm{M}$ Z-VAD-FMK, in order to block caspases' activity and facilitate the recovery of the Ripoptosome, as described in Tenev et al. [8]. A positive control for Ripoptosome assembly was added in the co-immunoprecipitation experiments depicted in figure B, where HEK293T were treated for 24 hours with $100 \mu \mathrm{M}$ Etoposide and $20 \mu \mathrm{M}$ Z-VAD-FMK. A) 2 milligrams of lysates from either empty vector (E.V.) or ZFP36 transfected cells (ZFP36) were loaded on a Sepharose column and fractions were eluted at defined timing and then loaded on polyacrylamide gel to perform Western blotting. In the presence of ZFP36, RIP1 was detected in higher molecular weight fractions (54-71), while this was less evident in E.V. samples. A positive control for RIP1 was loaded for each sample series $(\mathrm{C}+$ ). Molecular weight markers were eluted through the Sepharose column in order to identify the $2 \mathrm{MDa}$ fractions. B) Ripoptosome recovery was carried out by immunoprecipitating caspase8, while immunoprecipitation with nonspecific mouse IgG was performed on the Etoposide (Eto.) sample as a technical control for the procedure. Both in the presence of ZFP36 or Etoposide (Eto.) RIP1 was associated with caspase8, while this was less evident in the empty vector population (E.V.). Higher levels of FADD were associated to caspase8 in the empty vector population, as opposite to the ZFP36 and Etoposide (Eto.) samples. Input controls are represented on the right panel. C, D) Image J software provided a relative quantification of the levels of RIP1 and FADD associated to caspase8. 


\section{ZFP36-mediated RIP1 stabilization occurs in glioma neural stem cell lines}

To test the relevance of our data, we assessed the ability of ZFP36 to control RIP1 stability in human glioma neural stem cell lines (GNS), as this population shows resistance to standard therapy and is believed to mediate tumor recurrence [13]. A panel of six different glioblastomaderived GNS cell lines were screened for ZFP36 expression, which resulted to be very heterogeneous (data not shown). G144 GNS [18] express both ZFP36 and RIP1 when cultured in proliferation medium, and we detected a reduction in the total RIP1 levels (Figure 3) upon depletion of ZFP36, thus demonstrating that ZFP36 controls RIP1 stability in this cancer stem cell context.

\section{Discussion}

RIP1 is a cytosolic molecule capable of mediating either NF-kB signalling in resting conditions or necroptotic and apoptotic cell death in response to stress [19]. The regulation of RIP1 activity is dependent on the IAP E3 ubiquitine ligases cIAP-1, cIAP-2 and XIAP, which constitutively ubiquitinate RIP1 in transformed cells, where K63 ubiquitination promotes the assembly of a prosurvival complex [19], while K48 might mediate RIP1 proteasomal degradation [8]. Anticancer drugs such as Etoposide and SMAC-mimetics induce loss of IAPs and promote Ripoptosome assembly, while some natural molecules such as Interferons, are able to promote
RIP1-dependent necroptosis [11]. A recent report demonstrated that Etoposide induces ZFP36 expression [20] and, moreover, it is interesting to note that ZFP36 levels rise in response to the "breast cancer susceptibility protein" BRCA1 [21], which is implicated in the DNA damage response. Even though Interferons induce ZFP36 expression [22], it is still unknown whether ZFP36 is involved in mediating necroptosis in this setting.

Our data show that the ZFP36 mRNA binding protein regulates negatively the stability of XIAP in glioma cancer cells [4] and here we demonstrate in HEK293T cells that ZFP36-dependent destabilization of cIAP2 and XIAP leads to an increase in RIP1 levels and favors RIP1 association to caspase- 8 and FADD, while inducing cell death. These observations are consistent with described NF-kB inhibitory activity of ZFP36 [23] and with reports of synergism between ZFP36 and TNF alpha in inducing cell death in HEK293 cells [6]. Since our approach relies on ZFP36 restoration through transfection and is dependent on E3 ubiquitine ligases depletion, we identify the described molecular complex as the Ripoptosome. However, we cannot exclude that ZFP36 could mediate the association of RIP1, caspase- 8 and FADD also in response to death receptor signaling, as ZFP36 is induced and post-translationally regulated in response to TNF receptor activation [24, 25].

The observed control of ZFP36 over RIP1 stability in GNS cell lines further supports the molecular mechanism here described and suggests that GNS cell lines

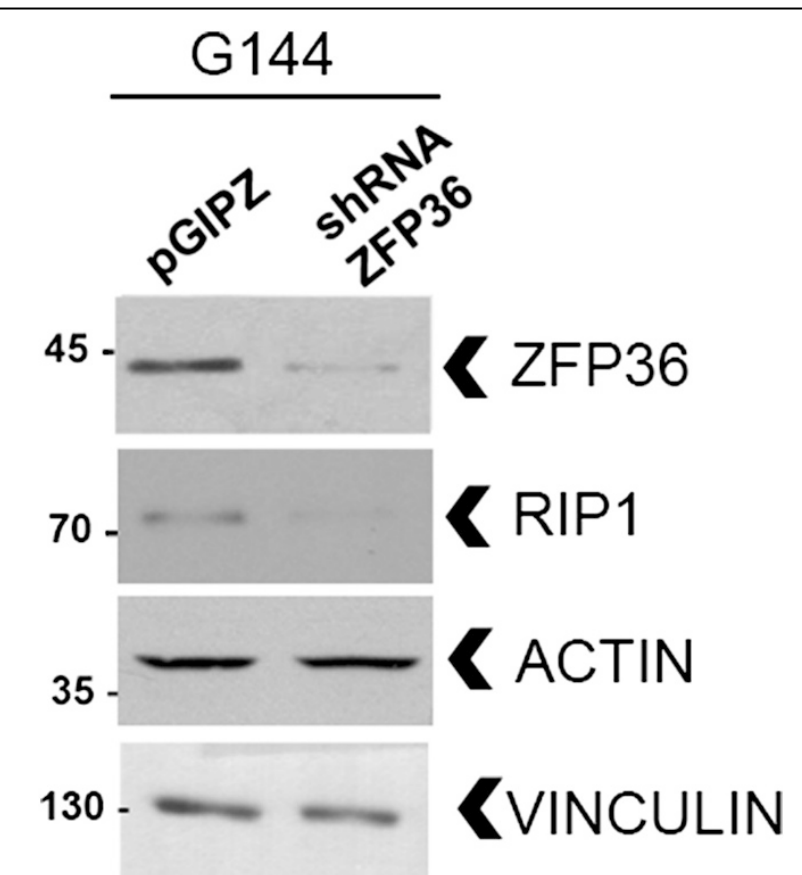

Figure 3 ZFP36 controls RIP1 stability in glioma neural stem cells. Human G144 glioma neural stem cells were grown in adhesion in proliferation medium and transduced with a short hairpin lentivirus directed against ZFP36 (shZFP36) or with a scrambled short hairpin sequence (pGIPZ) and sorted based on eGFP-positivity. Western blot analysis suggests that RIP1 stability is reduced in the absence of ZFP36 (shZFP36) when compared to G144 that retain ZFP36 expression (pGIPZ). 
that loose ZFP36 expression may be less sensitive to necroptotic-inducing treatments. Taken together, our results demonstrate a novel role for ZFP36 in regulating Ripoptosome assembly and RIP1-dependent necroptosis through IAP depletion.

\section{Additional file}

\section{Additional file 1: ZFP36 controls RIP1 stability by regulating IAPs.} The expression of ZFP36 in HEK293 (pRRL-ZFP36) with a lentiviral vector increases the levels of RIP1, when compared to the empty pRRL vector (pRRL). This effect is restrained by the co-transfection of a XIAP expression construct depleted of its own 3'UTR (pRRL-ZFP36 + D 3'UTR XIAP).

\section{Abbreviations}

ZFP36: Zinc finger protein 36 homolog; E.V: Empty vector; Eto: Etoposide; Nec-1: Necrostatin; IAPs: Inhibitor of Apoptosis Proteins; GNS: Glioma neural stem; TTP: Tristetraprolin; AU-rich: Adenine Uridine-rich; TNF alpha: Tumor necrosis factor alpha; RIP1: Receptor interacting serine/threonine protein kinase 1; FADD: Fas associated-protein with death domain; TRAF2: TNF receptor-associated factor 2; CIAP2: Cellular inhibitor of apoptosis 2; XIAP: X-linked inhibitor of apoptosis; 3'UTR: 3'untranslated region; P.I: Propidium lodide; MDa: Mega Dalton; DMSO: Dimethyl sulfoxide; MLKL: Mixed lineage kinase domain-like.

\section{Competing interests}

The authors declare that they have no competing interests.

\section{Authors' contributions}

TZM and TS conceived the study and wrote the manuscript, TS performed overexpression and silencing experiments, immunoprecipitations, fractioning and cell death assays. CA and MdA performed overexpression experiments, cell detah assays and GPCRs. CA, MdA and NV participated to fractioning experiments. LM cloned the ZFP36 cds in the pRRL lentivector. PS provided the $\triangle 3^{\prime}$ UTR XIAP construct, the CIAP2 and FADD antibodies, the G144 cell line. PS, LM, AM, FG, NV, SP, SF and AG contributed to data interpretation. All the authors read and approved the final manuscript.

\section{Authors' information}

Alexis Grande and Tommaso Zanocco-Marani are shared last author.

\section{Acknowledgments}

The authors acknowledge Domenico D'Arca and Massimo Ganassi at the University of Modena and Reggio Emilia for useful discussions. TS was supported by an AlL fellowship (Associazione Italiana contro le leucemielinfomi e mieloma).

\section{Author details}

'Department of Life Sciences, University of Modena and Reggio Emilia, Modena 41125, Italy. ${ }^{2}$ Samantha Dickson Brain Cancer Unit, UCL Cancer Institute, London WC1E 6BT, United Kingdom.

Received: 15 May 2014 Accepted: 28 April 2015

Published online: 06 May 2015

\section{References}

1. Sanduja S, Blanco FF, Young LE, Kaza V, Dixon DA. The role of tristetraprolin in cancer and inflammation. Front Biosci. 2012;17:174-88.

2. Stoecklin G, Gross B, Ming X-F, Moroni C. A novel mechanism of tumor suppression by destabilizing AU-rich growth factor mRNA. Oncogene. 2003;22:3554-61.

3. Al-Souhibani N, Al-Ahmadi W, Hesketh JE, Blackshear PJ, Khabar KSA. The RNA-binding zinc-finger protein tristetraprolin regulates AU-rich mRNAs involved in breast cancer-related processes. Oncogene. 2010;29:4205-15.

4. Selmi T, Martello A, Vignudelli T, Ferrari E, Grande A, Gemelli C, et al. ZFP36 expression impairs glioblastoma cell lines viability and invasiveness by targeting multiple signal transduction pathways. Cell Cycle. 2012;11(10):977-87.
5. Rounbehler RJ, Fallahi M, Yang C, Steeves MA, Li W, Doherty JR, et al. Tristetraprolin impairs myc-induced lymphoma and abolishes the malignant state. Cell. 2012;150:563-74.

6. Johnson BA, Blackwell TK. Multiple tristetraprolin sequence domains required to induce apoptosis and modulate responses to TNFalpha through distinct pathways. Oncogene. 2002;21:4237-46.

7. Kroemer G, Galluzzi L, Vandenabeele P, Abrams J, Alnemri ES, Baehrecke EH, et al. Classification of cell death: recommendations of the Nomenclature Committee on Cell Death 2009. Cell Death Differ. 2009;16:3-11.

8. Tenev T, Bianchi K, Darding M, Broemer M, Langlais C, Wallberg F, et al. The ripoptosome, a signaling platform that assembles in response to genotoxic stress and loss of IAPs. Mol Cell. 2011;43:432-48.

9. Feoktistova M, Geserick P, Kellert B, Dimitrova DP, Langlais C, Hupe M, et al. CIAPs Block Ripoptosome Formation, a RIP1/Caspase-8 Containing Intracellular Cell Death Complex Differentially Regulated by cFLIP Isoforms. Mol Cell. 2011;43:449-63.

10. Thapa RJ, Basagoudanavar SH, Nogusa S, Irrinki K, Mallilankaraman K, Slifker MJ, et al. NF-kB protects cells from gamma interferon-induced RIP1-dependent necroptosis. Mol Cell Biol. 2011;31:2934-46.

11. Thapa RJ, Nogusa S, Chen P, Maki JL, Lerro A, Andrake M, et al. Interferon-induced RIP1/RIP3-mediated necrosis requires PKR and is licensed by FADD and caspases. Proc Natl Acad Sci U S A. 2013;110:E3109-18.

12. Kim CW, Kim HK, Vo M-T, Lee HH, Kim HJ, Min YJ, et al. Tristetraprolin controls the stability of CIAP2 mRNA through binding to the 3'UTR of CIAP2 mRNA. Biochem Biophys Res Commun. 2010;400(1):46-52.

13. Goffart N, Kroonen J, Rogister B. Glioblastoma-initiating cells: relationship with neural stem cells and the micro-environment. Cancers. 2013;5:1049-71.

14. Bertrand MJM, Milutinovic S, Dickson KM, Ho WC, Boudreault A, Durkin J, et al. CIAP1 and CIAP2 facilitate cancer cell survival by functioning as E3 ligases that promote RIP1 ubiquitination. Mol Cell. 2008;30:689-700.

15. Wang H, Sun L, Su L, Rizo J, Liu L, Wang LF, et al. Mixed lineage kinase domain-like protein MLKL causes necrotic membrane disruption upon phosphorylation by RIP3. Mol Cell. 2014;54(1):133-46.

16. Zhang H, Zhou X, McQuade T, Li J, Chan FK-M, Zhang J. Functional complementation between FADD and RIP1 in embryos and lymphocytes. Nature. 2011:471:373-6.

17. Lee E-W, Seo J-H, Jeong M-H, Lee S-S, Song J-W. The roles of FADD in extrinsic apoptosis and necroptosis. BMB Rep. 2012:45:496-508.

18. Pollard SM, Yoshikawa K, Clarke ID, Danovi D, Stricker S, Russell R, et al. Glioma stem cell lines expanded in adherent culture have tumor-specific phenotypes and are suitable for chemical and genetic screens. Cell Stem Cell. 2009:4:568-80.

19. Festjens N, Vanden Berghe T, Cornelis S, Vandenabeele P. RIP1, a kinase on the crossroads of a cell's decision to live or die. Cell Death Differ. 2007:14:400-10.

20. Lee JY, Kim HJ, Yoon NA, Lee WH, Min YJ, Ko BK, et al. Tumor suppressor p53 plays a key role in induction of both tristetraprolin and let-7 in human cancer cells. Nucleic Acids Res. 2013;41 (11):5614-25.

21. Harkin DP, Bean JM, Miklos D, Song YH, Truong VB, Englert C, et al. Induction of GADD45 and JNK/SAPK-dependent apoptosis following inducible expression of BRCA1. Cell. 1999;97:575-86.

22. Sauer I, Schaljo B, Vogl C, Gattermeier I, Kolbe T, Müller M, et al. Interferons limit inflammatory responses by induction of tristetraprolin. Blood. 2006:107:4790-7.

23. Schichl YM, Resch U, Hofer-Warbinek R, de Martin R. Tristetraprolin impairs NF-kappaB/p65 nuclear translocation. J Biol Chem. 2009:284:29571-81.

24. Carballo E. Roles of tumor necrosis factor-alpha receptor subtypes in the pathogenesis of the tristetraprolin-deficiency syndrome. Blood. 2001;98:2389-95

25. Schichl YM, Resch U, Lemberger CE, Stichlberger D, de Martin R, et al. Novel phosphorylation-dependent Ubiquitination of Tristetraprolin by MEK kinase 1 (MEKK1) and TNF-receptor associated factor 2 (TRAF2). J Biol Chem. 2011;286(44):38466-77. 